\title{
The Spinal Cord Ability Ruler (SCAR): combining aspects of two widely-used outcome measures into one
}

\author{
L. A. Harvey ${ }^{1}$ \\ ๑) International Spinal Cord Society 2018
}

This edition of Spinal Cord contains some interesting correspondence about a newly proposed outcome measure called "The Spinal Cord Ability Ruler" (SCAR) [1]. The SCAR builds on the years of work of others-it does not try to reinvent the wheel. It uses select items from the upper limb motor assessments of the International Standards for the Neurological Classification of Spinal Cord Injury (ISNCSCI) and the Spinal Cord Independence Measure (SCIM) [2] with a newly proposed scoring system. Specifically, it contains the right and left strength scores for $\mathrm{C} 5-\mathrm{C} 8$, and 16 items from the SCIM. The existing 6-point manual muscle test used in the ISNCSCI is collapsed into a 3-point score, and each of the included SCIM items is scored on a 4-point scale. A formula is used to derive an overall score of 100 points (see p.735 for details [1]).

The rational for the choice of items in the SCAR is intriguing and centres around the desire to create a clinical endpoint for clinical trials that reflects "volitional performance" and can be reliably measured. "Volitional performance" is defined by the authors as "voluntary taskspecific physical actions contributing to independence in ADLs" (p.731) [1]. For this reason, the bladder, bowel and respiratory items of the SCIM are excluded because they are largely controlled by the autonomic nervous system. The authors argue for the addition of a select few strength assessments of the upper limb to capture "volitional performance" in those with high cervical lesions on the basis that the relevant SCIM items for this group of individuals cannot be adequately tested early after injury.

The proposed scoring system certainly reduces complexity, particularly for the scoring of some of the SCIM items. There is inherent weighting in the total score by the inclusion of various items (for example the right and left strength scores for C5-C8) and by the use of a 3-point scoring system for the upper limbs muscles and a 4-point scoring system for the SCIM items. This is invariably the case when combining multiple ordinal scales into one score. However the scale has been scrutinised with Rasch Analyses which showed that the derived SCAR scale is both linear and hierarchical with a good spread of scores across a large sample of participants with spinal cord injury. Unlike many scales, a one point difference at one end of the SCAR scale is equivalent to a one point difference at the other end of the scale. The authors make a compelling argument about the value of using an outcome measure with a linear scale as a trial endpoint.

\footnotetext{
$\triangle$ L. A. Harvey

spinalcord@iscos.org.uk

1 University of Sydney, Sydney, Australia
}

The SCAR was developed and tested using the European Multicenter Study about Spinal Cord Injury (EMSCI) database. On three occasions a large random sample of observations was drawn from the database to test, develop and finally refine the model. This is a wonderful example of how data registries can be used. Of course this needs to be done with care because there is always the risk of over-fitting any model when relying on a retrospective fit to available data from one source. It would be nice to see the process repeated on a different dataset or on data from clinical trials.

One of the real appeals of the SCAR is that it can be used retrospectively on data that have already been collected because the revised scores can be derived from the original scores. A limitation of the SCAR, which the authors go to great length to explain, is the problem of dealing with people with central cord syndromes whose patterns of motor loss do not follow the usual rostral to caudal sequence. This issue is yet to be resolved and suggests that the SCAR needs to be used cautiously in clinical trials involving people with suspected central cord syndromes.

Interested readers are encouraged to read a letter to the Editor in this edition about the SCAR paper [3]. The first author is Professor Catz, the original author of the SCIM [4]. The authors of the SCAR paper have responded to the issues raised by Professor Catz and his colleagues in a rebuttal letter [5]. Spinal Cord encourages exchanges such as these in which issues are debated in a respectful way.

The SCAR raises many interesting issues that help advance clinical trials involving people with spinal cord injury, so we welcome further discussion about the scale and its use, and we encourage all readers to take a close look at this freely-available paper.

\section{References}

1. Reed R, Mehra M, Kirshblum S, Maier D, Lammertse D, Blight A, et al. Spinal cord ability ruler: an interval scale to measure volitional performance after spinal cord injury. Spinal Cord. 2017;55:730-8.

2. Catz A, Itzkovich M, Tesio L, Biering-Sørensen F, Weeks C, Laramee MT, et al. A multicenter international study on the Spinal Cord Independence Measure, version III: Rasch psychometric validation. Spinal Cord. 2007;45:275-91.

3. Catz A, Tesio L, Benjamini Y. Comments on the Spinal Cord Ability Ruler. Spinal Cord. 2018;56:523-4.

4. Catz A, Itzkovich M, Agranov E, Ring H, Tamir A. SCIM-spinal cord independence measure: a new disability scale for patients with spinal cord lesions. Spinal Cord. 1997;35:850-6.

5. Steeves J, Curt A, Mehra M, Rupp R, Blight A, Maier D et al. The Spinal Cord Ability Ruler (SCAR) complements the Spinal Cord Independence Measure (SCIM). Spinal Cord. 2018;56:525-6. 